\title{
LA INFLUENCIA DE LOS RESULTADOS ECONÓMICOS SOBRE EL APOYO ELECTORAL DE LOS GOBIERNOS AUTONÓMICOS, 1980-1997
}

\author{
Ignacio Lago Peñas \\ Centro de Estudios Avanzados en Ciencias Sociales \\ E-mail: ilago@ceacs.march.es \\ Santiago Lago Peñas \\ Universidad de Santiago de Compostela \\ E-mail: easlago@usc.es
}

\begin{abstract}
RESUMEN
Este artículo estudia la validez de la incumbency-oriented hypothesis en las Comunidades Autónomas españolas. Los resultados de nuestro análisis sugieren que los factores políticos resultan más relevantes que la evolución de las variables económicas y presupuestarias en la explicación y predicción del apoyo electoral de los partidos en el gobierno.
\end{abstract}

\section{INTRODUCCIÓN ${ }^{1}$}

En la vasta literatura sobre el comportamiento electoral de los españoles publicada en los últimos veinte años en nuestro país (Montero y Pallarés, 1992), la relación entre la evolución económica general y las preferencias políticas de los electores apenas ha sido investigada. El limitado desarrollo de este tipo de estudios y, en general, de los modelos espaciales del voto en la ciencia política europea, y por inclusión en la española, contrasta con su notable arraigo en los Estados Unidos y Gran Bretaña ${ }^{2}$.

${ }^{1}$ Agradecemos los comentarios de Luis Caramés, Ramón Máiz, Guillermo Márquez, José Ramón Montero y José Luis Raymond; así como la ayuda de María Cernadas en el tratamiento informático de los datos. La responsabilidad de cualquier error u omisión es, por supuesto, sólo nuestra.

${ }^{2}$ Los modelos espaciales del voto se basan en la premisa de que el comportamiento de los

\section{Reis}


Las causas de este desigual tratamiento debemos buscarlas prioritariamente en los propios requisitos políticos que hacen posible que las condiciones macroeconómicas afecten al respaldo electoral de los partidos: i) el electorado debe ser capaz de imputar al partido de gobierno la responsabilidad de la situación económica que atraviesa el país; ii) la oposición ha de tener una posibilidad real de alcanzar el poder; y iii) la distancia ideológica entre los partidos debe ser lo suficientemente reducida para que cada uno de ellos pueda erigirse como una alternativa creíble de gobierno.

Estos tres requisitos encajan mucho mejor en los sistemas bipartidistas norteamericano o inglés que en los sistemas multipartidistas europeos más o menos polarizados, lo que exige una revisión del modelo de análisis en este segundo caso. $\mathrm{Y}$ es que su capacidad explicativa y predictiva se reduce significativamente, en el sentido de que la influencia de las condiciones macroeconómicas sobre los resultados electorales puede ser señalada respecto a los partidos individuales, pero poco se puede decir sobre la transferencia de votos del partido de gobierno a los de oposición (Belucci, 1984: 389-390).

En este marco, los estudios acerca del impacto de las condiciones macroeconómicas sobre las preferencias políticas de los electorados aparecen guiados por dos grandes hipótesis: la incumbency-oriented hypothesis y la policy-oriented hypothesis ${ }^{3}$ (Çarcoğlu, 1995; Powell y Whitten, 1993; Whitten y Palmer, 1999).

La primera de ellas resulta la más sencilla y la más extendida: las condiciones económicas tienen un efecto uniforme sobre el respaldo electoral de los partidos responsables de las mismas. Es decir, los votantes no tienen en cuenta la ideología del gobierno cuando evalúan los resultados macroeconómicos de su gestión. La prosperidad económica aparece así indefectiblemente ligada a un aumento del apoyo electoral del partido de gobierno en los comicios siguientes. Por el contrario, una coyuntura económica desfavorable penaliza electoralmente al partido de gobierno en favor de la oposición ${ }^{4}$.

De forma más general, esta hipótesis se enriquece con la inclusión de variables explicativas adicionales de carácter presupuestario. Las razones para tal ampliación son varias. En primer lugar, la dinámica del empleo, la renta y los precios depende sólo parcialmente de la gestión de los gobiernos; lo que resulta aún más evidente al descender de ámbito territorial. En este sentido, las varia-

votantes y los candidatos está guiado por el self-interest: los votantes comparan el programa de cada partido o candidato y eligen el que consideran más favorable para sus propias preferencias, que, por supuesto, no se refieren exclusivamente a la renta económica.

${ }^{3}$ La literatura sobre la influencia de las fluctuaciones macroeconómicas sobre los electores abarca también el estudio de los efectos de las políticas llevadas cabo por los diferentes partidos y el análisis de los ciclos político-electorales, esto es, el comportamiento oportunista de los partidos que manipulan la economía para incrementar sus posibilidades de reelección (Alesina, Londregan y Rosenthal, 1993).

${ }_{4}^{4}$ Vid., entre el amplísimo número de investigaciones existentes, los surveys iniciales de Monroe (1979) y Paldam (1981), y los estudios de Alesina, Londregan y Rosenthal (1993), Bellucci (1984), Erikson (1989), Leithner (1991) y Lewis-Beck y Bellucci (1982). 
bles presupuestarias son susceptibles de un control más directo por parte de los ejecutivos y están sujetas a evaluación por parte de los electores. La utilización electoralista de los recursos públicos, que bien pudiera responder a una pauta cíclica regida por el calendario electoral, emerge entonces como una posibilidad a tener en cuenta (Blaos y Nadeau, 1992; Díaz de Serralde, 1999; Lago, 1997; Sanjiao, 1991).

En segundo lugar, existen otros factores adicionales potencialmente relevantes en el análisis de gobiernos subcentrales integrados en estructuras fiscales de tipo federal. Por un lado, la coincidencia entre el color político de los partidos de gobierno central y subcentral podría tener una incidencia real o supuesta en el reparto territorial de fondos públicos susceptibles de empleo discrecional por el primero - inversiones estatales, por ejemplo-, lo que influiría sobre las decisiones de los votantes. Por otro, el potencial electoralista de las decisiones financieras de un gobierno depende de forma directa de su tamaño presupuestario.

Por su parte, la policy-oriented hypothesis reconoce diferencias en las nociones manejadas por los votantes acerca de la competencia y prioridades de los partidos en la gestión de diferentes problemas económicos. Los electores apoyarán así a aquellas fuerzas políticas que consideran más capaces de resolver los problemas económicos puntuales por los que atraviesa un país. Las diferentes situaciones económicas tienen, pues, distintos efectos sobre los resultados electorales de los partidos según su ideología 5 .

Dada la indisponibilidad de datos para estudiar este último caso ${ }^{6}$, el presente trabajo contrasta la incumbency-oriented hypothesis en las elecciones autonómicas celebradas en España desde 1980 hasta 1997. Nuestro objetivo es comprobar si efectivamente la evolución macroeconómica y presupuestaria en el ámbito autonómico ha incidido en el apoyo electoral de los partidos de gobierno. La investigación tiene, por tanto, un carácter comparado entre las diecisiete Comunidades Autónomas y, consecuentemente, está basada en la aplicación de un análisis de regresión de sección cruzada. En todo caso, las características de las arenas político-electorales autonómicas - multipartidismo, gobiernos de coalición en bastantes casos y un elevado grado de nacionalización del comportamiento electoral- y las especificidades institucionales del caso español reclaman la adaptación del modelo de análisis y una labor previa de selección de observaciones válidas.

5 La bibliografía sobre esta cuestión también es enorme. Vid., por ejemplo, los surveys de Alesina (1988), Alesina y Rosenthal (1995) y Hibbs (1992), y los estudios de Boix (1996), Çarcoğlu (1995), Powell y Whitten (1993) y Whitten y Palmer (1999).

${ }^{6}$ Esta indisponibilidad de datos no significa que la policy-oriented hypothesis no sea relevante en España. Por el contrario, para Maravall y Fraile (1998: 25 ss.), por ejemplo, la explicación de la escasa influencia de los elevados niveles de desempleo en el respaldo electoral del PSOE en las elecciones generales residiría precisamente en la creencia de una buena parte de la población de que otro partido no mejoraría la actuación del gobierno. 


\section{UN MODELO PARA EL ANÁLISIS EMPÍRICO}

En las dos décadas de funcionamiento del Estado de las Autonomías en España se ha desarrollado una serie significativa de procesos electorales de ámbito autonómico, lo que posibilita la realización de análisis longitudinales. Las Comunidades Autónomas del artículo 151 de la Constitución (País Vasco, Andalucía, Galicia y Cataluña), que cuentan con capacidad de convocatoria electoral propia, han celebrado cinco elecciones cada una; excepto Euskadi, que suma ya seis. Por su parte, Navarra y las Comunidades de la llamada vía «normal» del artículo 143 mantienen un proceso político-electoral coincidente en el tiempo: todas han agotado cuatro legislaturas.

La contrastación de la incumbency-oriented hypothesis para las elecciones autonómicas se realiza inicialmente sobre las 72 convocatorias electorales de tal carácter celebradas entre 1980 y 1997. Los comicios vascos de octubre de 1998 no se incluyen en el análisis por la indisponibilidad de los datos económicopresupuestarios.

La variable dependiente es el incremento en el porcentaje de votos del partido o coalición de gobierno autonómico sobre el total de sufragios en dos elecciones consecutivas $(\Delta \mathrm{V})$. En el caso de las coaliciones, el porcentaje de voto total de las mismas es igual a la suma de los porcentajes de cada uno de sus componentes. Los partidos o coaliciones de gobierno son aquellos que han desempeñado las tareas de gobierno en exclusiva durante una legislatura, haya sido ésta agotada o no. De este modo, se excluyen del análisis todos los casos en los que ha habido cambios en las fuerzas políticas que componen los gobiernos autonómicos durante la legislatura. Nos referimos a las Comunidades Autónomas de Aragón, en la tercera legislatura; Canarias, en la segunda y la tercera; Cantabria, en la segunda; Galicia, en la segunda; País Vasco, en la cuarta; y La Rioja, en la segunda ${ }^{7}$. Por el contrario, las remodelaciones de los gobiernos que se han limitado a cambios en los titulares de las consejerías o en la Presidencia autonómica no han sido tenidas en cuenta, en tanto las políticas partidistas llevadas a cabo no deberían sufrir modificaciones de importancia. La fuente de los resultados electorales es la Subdirección General de Política Interior y Procesos Electorales del Ministerio del Interior.

Las variables independientes que se recogen en el modelo son de carácter económico-presupuestario y político-electoral. Así, además de incluir como variables explicativas las habituales en la literatura — renta per cápita y tasa de desempleo-, incorporamos dos de naturaleza presupuestaria: la inversión pública en infraestructuras sociales y productivas y la deuda pública autonómica.

La lógica que subyace a la elección de estos cuatro factores es la siguiente. En el caso de la renta per cápita y del paro, es razonable pensar que el grado de popularidad de los gobiernos aumente cuanto más favorable sea el comporta-

7 Vid. Alcántara y Martínez (1998), Llera (1998), Pallarés (1995), Pallarés, Montero y Llera (1997) y Wert (1998). 
miento de la producción y el empleo, y viceversa. Aunque ciertamente la enfurtida red tejida por las relaciones económicas interregionales provoca que las dinámicas individuales se encuentren estrechamente ligadas a lo que ocurre en el conjunto del país (Raymond, 1994), existiría un cierto margen para los comportamientos diferenciales — potenciales y efectivos - impulsados desde la propia Comunidad Autónoma. No parece que ocurra lo mismo con la tasa de inflación, variable que depende de un modo crucial de las decisiones monetarias que se adoptan en el ámbito estatal y que, en cualquier caso, hasta hace bien poco generaba escasa preocupación entre la ciudadanía española (Fuentes y Alcaide, 1995). Por consiguiente, y aunque su inclusión es una fórmula habitual en la literatura, optamos por no tener en consideración el comportamiento de los precios.

En cuanto a las variables de naturaleza presupuestaria, la justificación de su inclusión en el modelo descansa en razones diferentes. En el caso de la deuda, cabe contemplarla como un instrumento a disposición de los gobiernos autonómicos para incrementar la cantidad y calidad de los bienes y servicios públicos, con un coste político inferior que el que comporta la financiación impositiva. Siempre, eso sí, que existan fenómenos de «ilusión financiera», que distorsionan la percepción de los ciudadanos de sus facturas fiscales presentes y futuras (Suárez Pandiello, 1996), o marcos institucionales que posibiliten la traslación de la deuda entre los niveles de la Hacienda, bajo el manto de acuerdos de asunción de pasivos financieros.

Por lo que respecta a la inversión pública en infraestructuras, su dinámica es importante, en tanto influye positivamente sobre el crecimiento económico - vía efectos de demanda, vía incremento de la productividad o vía atracción de factores productivos privados- y es utilizada como bien de consumo final por los ciudadanos. Aunque, por supuesto, la actividad financiera desarrollada por los sectores públicos autonómicos es mucho más completa y, por tanto, cabría de entrada incorporar otras variables explicativas, las peculiaridades institucionales del ámbito analizado aconsejan ser cautos al respecto. En efecto, los años que comprende el período muestral han sido testigos de un vigoroso proceso de descentralización política y financiera llevado a cabo a diferentes ritmos según Comunidades y momentos, y que ha dado lugar a panoramas presupuestarios muy distintos entre años electorales sucesivos. Adicionalmente, los problemas de corresponsabilidad fiscal y la convivencia de administraciones con competencias muchas veces superpuestas —el caso de la red viaria sería un ejemplo paradigmático - dificultarían la vinculación entre cada uno de los bienes y servicios públicos y la respectiva administración competente.

La operacionalización de estas cuatro variables económico-presupuestarias responde a diferentes estrategias. En primer lugar, la renta per cápita (RPC) la definimos como el cociente entre el valor añadido bruto a coste de los factores (VAB c. f.) y la población. Los datos han sido tomados, en febrero de 1999, de la base informatizada SOPHINET, auspiciada por la Fundación BBV ${ }^{8}$. Por su

${ }^{8}$ Localizada en la dirección de la red Internet http://bancoreg.fbbv.es. 
parte, la tasa de paro (U) procede de la encuesta de población activa (EPA) y la calculamos como media anual simple de los resultados trimestrales. La cuantificación de los niveles de la deuda pública autonómica (DP) se realiza a partir de los datos sobre pasivos no comerciales materializados en instrumentos financieros computados por el Banco de España para el período 1984-1997. Para los años anteriores a 1983 hemos supuesto un endeudamiento igual a cero y para 1983 hemos utilizado el correspondiente a 1984. Dada la escasa cuantía de la deuda acumulada en todas las Comunidades hasta 1984, y puesto que el stock de pasivos lo ponderamos por el VAB c. f. regional, podríamos aceptar este procedimiento de extrapolación como una aproximación razonable.

Por último, la inversión pública se define como el incremento del stock neto de capital público $(\mathrm{K})$ de cada una de las regiones materializado en infraestructuras productivas - carreteras, autopistas, ferrocarril, infraestructuras hidráulicas, puertos, aeropuertos y estructuras urbanas de las corporaciones locales - y sociales — infraestructuras educativas y sanitarias - . Los datos proceden de las series de capital público elaboradas por el Instituto Valenciano de Investigaciones Económicas (IVIE) y patrocinadas por la Fundación BBV. La información para el período 1980-1994 ha sido tomada de SOPHINET. En los casos en los que fue necesaria información para años posteriores, extrapolamos las tasas de crecimiento de los ejercicios inmediatamente anteriores.

Para la renta per cápita y el capital público utilizamos el porcentaje de incremento entre los años electorales anualizado linealmente ${ }^{9}$. En el caso de la tasa de paro y el ratio deuda/PIB, computamos el promedio anual de su variación entre período ${ }^{10}$.

El modelo se completa con la introducción de una variable de tipo político-electoral. Los procesos electorales autonómicos muestran un elevado grado de nacionalización del comportamiento, con tendencias paralelas de evolución electoral como expresión de la influencia determinante de factores de ámbito general (Llera, 1998; Pallarés, 1995). En consecuencia, consideramos conveniente incorporar la trayectoria de los apoyos electorales de los partidos de gobierno autonómicos en el nivel estatal. La variable que recoge esta evolución paralela es el incremento en el porcentaje de voto del partido o coalición de gobierno autonómico entre las dos elecciones generales que preceden a las convocatorias electorales autonómicas en cada caso (EG). El porcentaje de voto de los partidos nacionalistas o regionalistas que forman parte de los gobiernos autonómicos se computa en el ámbito de la Comunidad Autónoma; de otro modo no sería razonable cotejar sus porcentajes de sufragio con los correspondientes a las fuerzas políticas de implantación estatal. También en este caso, la fuente de los resultados electorales es la Subdirección General de Política Interior y Procesos Electorales del Ministerio del Interior.

9 Esto es, $(1 / k)^{*}\left[\ln \left(X_{t}\right)-\ln \left(X_{t-k}\right)\right]$, siendo $k$ el número de años transcurridos entre elecciones consecutivas.

${ }^{10}$ Es decir, $(1 / k)^{*}\left[X_{t}-X_{t-k}\right]$, con $k$ definido igual que en la nota anterior. 


\section{RESULTADOS DEL ANÁLISIS}

La tabla 1 presenta los coeficientes de correlación entre el incremento del apoyo electoral de los gobiernos autonómicos y las cinco variables independientes de carácter económico-presupuestario y político-electoral que, expresadas en variaciones, integran el modelo para el análisis empírico.

\section{TABLA 1}

Matriz de correlaciones*

\begin{tabular}{|c|c|c|c|c|c|c|}
\hline & $\Delta \mathrm{V}$ & $\triangle \mathrm{RPC}$ & $\Delta \mathrm{U}$ & $\Delta \mathrm{K}$ & $\Delta \mathrm{DP}$ & $\Delta \mathrm{EG}$ \\
\hline$\Delta \mathrm{V}$ & 1 & & & & & \\
\hline$\triangle \mathrm{RPC}$ & 0,04 & 1 & & & & \\
\hline$\Delta \mathrm{U}$ & $-0,22$ & $-0,42$ & 1 & & & \\
\hline$\Delta \mathrm{K}$ & 0,27 & $-0,02$ & $-0,21$ & 1 & & \\
\hline$\Delta \mathrm{DP}$ & $-0,01$ & $-0,33$ & 0,13 & 0,35 & 1 & \\
\hline$\Delta \mathrm{EG}$ & 0,41 & $-0,36$ & 0,27 & 0,11 & 0,22 & 1 \\
\hline
\end{tabular}

* $\Delta \mathrm{V}$ es el incremento del porcentaje de voto del partido/s de gobierno autonómico, $\Delta \mathrm{RPC}$ es el incremento de la renta per cápita, $\Delta \mathrm{U}$ es el incremento de la tasa de paro, $\Delta \mathrm{K}$ es el incremento en el stock neto de capital público, $\Delta \mathrm{DP}$ es el incremento de la deuda pública autonómica, y $\Delta \mathrm{EG}$ es el incremento del porcentaje de voto del partido/s de gobierno autonómico en las elecciones generales.

Sin duda, el resultado más sorprendente lo constituye la baja correlación entre las variables económico-presupuestarias y la dinámica del apoyo electoral del partido o coalición de gobierno autonómico; sobre todo en el caso de la renta per cápita, una de las variables más importantes en la literatura. Su escasa relevancia, además de su moderada correlación negativa con el paro, aconsejan su exclusión en las especificaciones econométricas para evitar problemas de multicolinealidad $^{11}$. Otro tanto ocurre con el incremento del ratio deuda/PIB, por lo que hemos optado por una solución similar. Así las cosas, seleccionamos una especificación econométrica en la que tan sólo aparecen como variables explicativas el capital público y la tasa de paro:

$$
\Delta V_{i t}=\beta_{0}+\beta_{1} \cdot \Delta K_{i t}+\beta_{2} \cdot \Delta U_{i t}+\varepsilon_{i t}
$$

donde $\varepsilon$ es la perturbación aleatoria y los subíndices $i$ y $t$ indican, respectivamente, la Comunidad y el año de las elecciones.

${ }^{11}$ La renta per cápita tampoco era relevante cuando se utilizaba únicamente la tasa de crecimiento correspondiente al segundo bienio de cada legislatura. 
La estimación econométrica de [1] presenta problemas de heterocedasticidad, si atendemos a los resultados del test de White (1980) aplicado sobre los residuos mínimos cuadráticos ordinarios (MCO). En consecuencia, hemos estimado la matriz de varianzas y covarianzas y, por consiguiente, el valor de los estadísticos-t de significatividad mediante el método propuesto por el propio White (1980), robusto ante la presencia de varianzas cambiantes ${ }^{12}$.

Los resultados se recogen en la tabla 2 (columna 1). El ajuste econométrico es deficiente: tan sólo el 10 por 100 de la varianza vendría determinado por el comportamiento de la inversión pública y el paro. Además, la significatividad de esta última variable es tan sólo marginal. Por otro lado, la inclusión de la renta per cápita y la deuda pública no mejoraba sustancialmente el ajuste.

Probamos también la incorporación de la coincidencia de color político entre el gobierno autonómico y estatal (CO). La correlación entre esta variable dicotómica y la variable explicada es elevada $(0,51)$, pero de signo negativo. Interpretado literalmente, esto significa que la coincidencia política de los gobiernos conllevaría un peor rendimiento electoral. La explicación de este resultado contraintuitivo se encuentra en la correlación estadística elevada y negativa existente entre la variable $\mathrm{CO}$ y la que recoge la trayectoria en las elecciones generales $(-0,71)$ : dado que el período analizado se caracteriza por la pérdida tendencial del apoyo al partido de gobierno en el ámbito estatal, la coincidencia de color político se produce respecto a un ejecutivo en declive electoral, situación que se proyecta a su vez sobre los resultados electorales en el ámbito autonómico.

Asimismo, optamos por incluir una variable dicotómica con valor 1 para las Comunidades Forales y del artículo 151 y asimiladas y 0 para las restantes, a fin de contrastar la idea de que un mayor tamaño presupuestario ayudaría a mantener los apoyos electorales. Sin embargo, su coeficiente de correlación lineal con la variable $\mathrm{V}$ era apenas del 0,10 y su significatividad estadística como regresor despreciable. Vistos estos resultados, nos planteamos la necesidad de incorporar como factor explicativo adicional el resultado de las elecciones generales:

12 Dadas las características de la base de datos utilizada, no nos pareció adecuado recurrir a una metodología econométrica más sofisticada, diseñada para el tratamiento de datos de panel (Greene, 1997). En primer lugar, la estrecha dimensión temporal de éste nos hace descartar la estimación de coeficientes individuales y, por tanto, la utilización de metodología $S U R E$ a fin de controlar correlaciones contemporáneas entre las perturbaciones correspondientes a las distintas ecuaciones. Por el mismo motivo, descartamos la estimación de matrices de varianzas-covarianzas individuales. Por lo que respecta a la consideración de efectos específicos individuales, renunciamos tanto al modelo de efectos fijos como al de efectos aleatorios. En el primer caso, por la pérdida de grados de libertad que suponían. En el segundo, por el carácter incompleto e irregular del panel disponible; sin olvidar que la validez de los efectos aleatorios depende de su incorrelación con el término de la perturbación y que, en el supuesto de que ésta sea asumible, los estimadores MCO serán igualmente consistentes. Finalmente, la consideración de efectos individuales dificultaría considerablemente el análisis de estabilidad estructural —-temporal y espacial— que realizamos más adelante. 


$$
\Delta V_{i t}=\beta_{o}+\beta_{I} \bullet \Delta K_{i t}+\beta_{2} \cdot \Delta U_{i t}+\beta_{3} \cdot \Delta E G_{i t}+\varepsilon_{i t}
$$

donde, al margen de las variables incluidas, los restantes elementos son los mismos que aparecen en la ecuación [1].

La estimación econométrica correspondiente aparece en la columna (2) de la tabla 2. El ajuste econométrico mejora notablemente, con un coeficiente de determinación de 0,34 . Destaca la elevada significatividad del nuevo regresor y el mantenimiento de los signos de $\mathrm{K}$ y $\mathrm{U}$; si bien se produce una inversión en el grado de significatividad relativa de ambas variables.

En cualquier caso, un análisis más detallado de los residuos, como el que se hace en la tabla 3, revela la existencia de observaciones atípicas o outliers que se generan errores importantes de predicción: Cataluña en 1984, Murcia en 1995 y País Vasco en 1986. Sin ánimo de exhaustividad, la anomalía de estas observaciones encuentra su explicación prioritariamente en términos políticos. En Cataluña, se trata de la primera y más importante manifestación del voto dual; en Murcia, se debe a la crisis de las "familias» del socialismo murciano, que llevó a la dimisión del Presidente de la Comunidad Autónoma poco antes de las elecciones generales de 1993; en el País Vasco, el motivo es la escisión de Eusko Alkartasuna (EA) del PNV en vísperas de los comicios autonómicos de 1986 - EA obtuvo más del 15 por 100 de los votos en esas elecciones-.

Al volver a estimar [2] excluyendo dichas observaciones, los resultados econométricos mejoran notablemente, amén de que dejan de presentar problemas de heterocedasticidad: el $\mathrm{R}^{2}$ aumenta de 0,34 a 0,47 y todas las variables pasan a ser significativas al nivel del 1 por 100 .

En resumen, estos resultados ponen en evidencia la dependencia de la dinámica electoral en el ámbito autonómico respecto a lo que acontece en el conjunto del país. Pero también demuestran que existen otros factores endógenos, distintos a los estrictamente económico-presupuestarios, cruciales para entender la dinámica del apoyo electoral a los partidos de gobierno.

A fin de contrastar la estabilidad estructural del modelo hemos vuelto a estimar las ecuaciones [1] y [2] empleando submuestras tanto espaciales como temporales. En el primer caso, tomamos las Comunidades Forales y del artículo 151 y asimiladas, por un lado, y las del artículo 143, por otro. En el segundo, distinguimos entre los procesos electorales celebrados en los años ochenta y los que tuvieron lugar en la década de los noventa. Las estimaciones las realizamos tanto con la muestra íntegra como excluyendo las observaciones anómalas antes reseñadas. Los resultados aparecen en las columnas (4) a (11) de la tabla 2.

Al comparar los ajustes correspondientes a las Comunidades Autónomas de competencias amplias [columna (4)] con las del artículo 143 [columna (6)] surgen panoramas bien diferentes. Mientras que para las primeras las variables de tipo económico-presupuestario serían irrelevantes, para las segundas aumentaría su significatividad estadística. Además, en este caso el crecimiento de la renta no genera problemas serios de multicolinealidad y su introducción mejora el ajuste. 
TABLA $2^{*}$

Efectos de las variables económico-presupuestarias y de las politico-electorales en el apoyo electoral de los partidos de gobierno autonómicos, 1980-1997**

\begin{tabular}{|c|c|c|c|c|c|c|c|c|c|c|c|}
\hline Variables & (1) & (2) & (3) & (4) & (5) & (6) & (7) & (8) & (9) & (10) & (11) \\
\hline Constante ....................... & $\begin{array}{l}-5,48 \\
(-2,31)\end{array}$ & $\begin{array}{l}-3,26 \\
(-1,36)\end{array}$ & $\begin{array}{l}-4,50 \\
(-2,29)\end{array}$ & $\begin{array}{l}-2,96 \\
(-0,52)\end{array}$ & $\begin{array}{l}-5,27 \\
(-1,36)\end{array}$ & $\begin{array}{l}-3,15 \\
(-1,26)\end{array}$ & $\begin{array}{l}-5,09 \\
(-1,78)\end{array}$ & $\begin{array}{l}-6,28 \\
(-4,19)\end{array}$ & $\begin{array}{l}-6,21 \\
(-3,10)\end{array}$ & $\begin{array}{l}-9,44 \\
(-2,09)\end{array}$ & $\begin{array}{l}-8,66 \\
(-1,89)\end{array}$ \\
\hline$\Delta \mathrm{K}$ & $\begin{array}{c}0,82 \\
(1,93)\end{array}$ & $\begin{array}{c}0,54 \\
(1,34)\end{array}$ & $\begin{array}{c}0,81 \\
(2,30)\end{array}$ & $\begin{array}{c}0,46 \\
(0,48)\end{array}$ & $\begin{array}{c}0,95 \\
(1,35)\end{array}$ & $\begin{array}{c}0,46 \\
(1,07)\end{array}$ & $\begin{array}{c}0,58 \\
(1,32)\end{array}$ & $\begin{array}{l}-0,10 \\
(-0,21)\end{array}$ & $\begin{array}{c}0,25 \\
(0,52)\end{array}$ & $\begin{array}{c}0,97 \\
(1,34)\end{array}$ & $\begin{array}{c}1,00 \\
(1,45)\end{array}$ \\
\hline$\Delta \mathrm{U} \ldots \ldots$ & $\begin{array}{l}-1,09 \\
(-1,21)\end{array}$ & $\begin{array}{l}-2,14 \\
(-2,66)\end{array}$ & $\begin{array}{c}-2,37 \\
(-3,61)\end{array}$ & $\begin{array}{l}-1,30 \\
(-1,03)\end{array}$ & $\begin{array}{l}-2,01 \\
(-2,02)\end{array}$ & $\begin{array}{l}-3,06 \\
(-3,07)\end{array}$ & $\begin{array}{l}-2,84 \\
(-2,95)\end{array}$ & $\begin{array}{c}2,63 \\
(1,74)\end{array}$ & $\begin{array}{c}1,09 \\
(0,95)\end{array}$ & $\begin{array}{l}-2,34 \\
(-2,50)\end{array}$ & $\begin{array}{l}-2,34 \\
(-2,55)\end{array}$ \\
\hline $\begin{array}{l}\Delta \mathrm{EG} \quad . . \\
\Delta \mathrm{RPC}\end{array}$ & & $\begin{array}{c}0,71 \\
(4,30)\end{array}$ & $\begin{array}{c}0,58 \\
(4,12)\end{array}$ & $\begin{array}{c}0,66 \\
(3,20)\end{array}$ & $\begin{array}{c}0,51 \\
(2,69)\end{array}$ & $\begin{array}{c}0,92 \\
(3,75) \\
1,12 \\
(1,67)\end{array}$ & $\begin{array}{c}0,85 \\
(3,05) \\
0,78 \\
(1,12)\end{array}$ & $\begin{array}{c}0,92 \\
(3,98)\end{array}$ & $\begin{array}{c}0,76 \\
(4,67)\end{array}$ & $\begin{array}{c}0,61 \\
(2,54) \\
1,64 \\
(1,94)\end{array}$ & $\begin{array}{c}0,55 \\
(2,72) \\
1,31 \\
(1,64)\end{array}$ \\
\hline $\mathrm{N}$ & 48 & 48 & 45 & 21 & 19 & 27 & 26 & 19 & 17 & 29 & 28 \\
\hline $\mathrm{R}^{2}$ & 0,10 & 0,34 & 0,47 & 0,28 & 0,46 & 0,52 & 0,51 & 0,60 & 0,66 & 0,55 & 0,52 \\
\hline
\end{tabular}

* $\Delta \mathrm{K}$ es el incremento en el stock neto de capital público, $\Delta \mathrm{U}$ es el incremento de la tasa de paro, $\Delta \mathrm{EG}$ es el incremento del porcentaje de voto del partido/s de gobierno autonómico en las elecciones generales, y $\triangle \mathrm{RPC}$ es el incremento de la renta per cápita.

** Aparecen en primer lugar los coeficientes de regresión, seguidos por los estadísticos t calculados a partir de la matriz de varianzas y covarianzas estimada mediante el método de mínimos cuadráticos ordinarios (MCO) en las columnas (5), (7), (9), (11), y mediante el método de White (1980) en las columnas (1), (2), (3), (4), (6), (8), (10). $\mathrm{R}^{2}$ es el coeficiente de determinación y $\mathrm{N}$ el número de observaciones.

(1) Todas las elecciones autonómicas.

(2) Todas las elecciones autonómicas.

(3) Todas las elecciones autonómicas sin outliers.

(4) Elecciones en las Comunidades Autónomas Forales y las del artículo 151.

(5) Elecciones en las Comunidades Autónomas Forales y las del artículo 151 sin outliers.

(6) Elecciones en las Comunidades Autónomas del artículo 143

(7) Elecciones en las Comunidades Autónomas del artículo 143 sin outliers.

(8) Elecciones en las Comunidades Autónomas en los años ochenta.

(9) Elecciones en las Comunidades Autónomas en los años ochenta sin outliers.

(10) Elecciones en las Comunidades Autónomas en los años noventa.

(11) Elecciones en las Comunidades Autónomas en los años noventa sin outliers. 


\section{TABLA 3}

Valores reales, valores estimados y residuos en la estimación (3) de la Tabla 2

\begin{tabular}{|c|c|c|c|}
\hline Elección & Valor real & Valor estimado & Residuo \\
\hline Andalucía 1986 & $-5,49$ & $-9,56$ & 4,07 \\
\hline 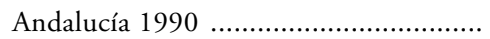 & 2,62 & 0,17 & 2,44 \\
\hline 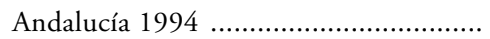 & $-10,78$ & $-5,52$ & $-5,25$ \\
\hline 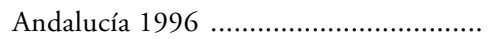 & 5,36 & 1,32 & 4,03 \\
\hline 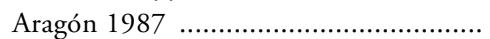 & $-10,89$ & $-5,86$ & $-5,02$ \\
\hline 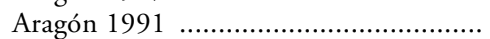 & $-3,47$ & 1,67 & $-5,14$ \\
\hline 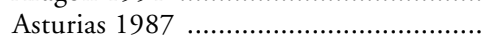 & $-12,84$ & $-8,40$ & $-4,43$ \\
\hline 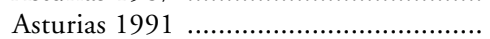 & 2,20 & $-1,38$ & 3,58 \\
\hline Asturias 1995 & $-7,29$ & $-3,00$ & $-4,28$ \\
\hline 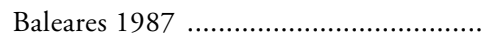 & 1,89 & $-0,47$ & 2,36 \\
\hline 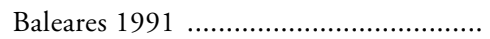 & 4,120 & 2,15 & 1,96 \\
\hline 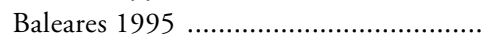 & 0,46 & 3,15 & $-2,69$ \\
\hline Canarias 1987 .................................................. & $-13,48$ & $-7,67$ & $-5,80$ \\
\hline 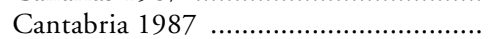 & $-1,56$ & $-5,21$ & 3,65 \\
\hline Cantabria 1995 & 1,28 & 3,39 & $-2,11$ \\
\hline Castilla-La Mancha 1987 ........................... & $-0,28$ & $-5,67$ & 5,39 \\
\hline 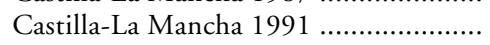 & 5,910 & $-2,19$ & 8,10 \\
\hline 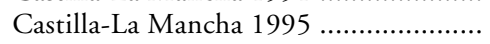 & $-6,52$ & $-5,50$ & $-1,01$ \\
\hline Castilla y León 1987 & $-10,25$ & $-6,05$ & $-4,19$ \\
\hline Castilla y León 1991 & 9,34 & 1,626 & 7,71 \\
\hline Castilla y León 1995 & 9,03 & 2,88 & 6,14 \\
\hline 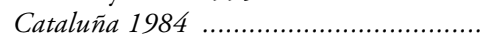 & 18,97 & $-3,28$ & 22,25 \\
\hline Cataluña 1988 & $-1,03$ & 6,62 & $-7,65$ \\
\hline 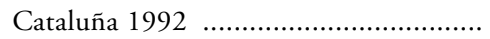 & 0,74 & 4,14 & $-3,40$ \\
\hline 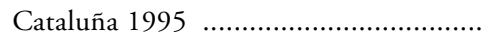 & $-5,39$ & $-4,56$ & $-0,82$ \\
\hline 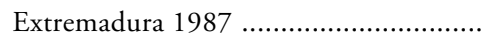 & $-3,68$ & $-9,17$ & 5,49 \\
\hline 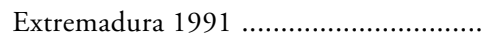 & 5,010 & $-1,11$ & 6,12 \\
\hline 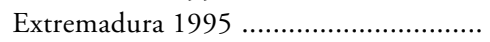 & $-10,32$ & $-3,67$ & $-6,64$ \\
\hline 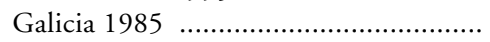 & 10,65 & 9,80 & 0,84 \\
\hline 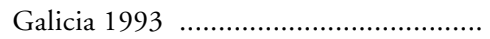 & 8,42 & 3,80 & 4,61 \\
\hline 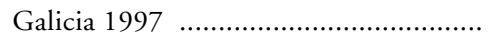 & 0,26 & 3,28 & $-3,02$ \\
\hline 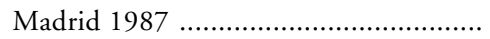 & $-11,76$ & $-4,37$ & $-7,38$ \\
\hline 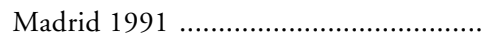 & $-2,05$ & $-0,22$ & $-1,82$ \\
\hline 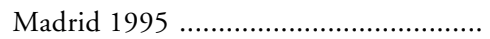 & $-6,84$ & $-5,96$ & $-0,87$ \\
\hline 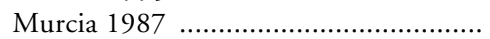 & $-8,77$ & $-4,21$ & $-4,55$ \\
\hline 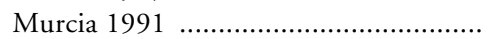 & 1,55 & $-1,42$ & 2,97 \\
\hline 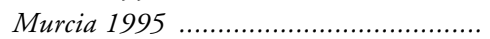 & $-13,60$ & $-3,56$ & $-10,03$ \\
\hline Navarra 1987 & $-7,79$ & $-6,07$ & $-1,71$ \\
\hline 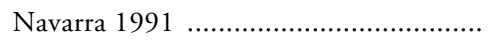 & 5,73 & $-0,38$ & 6,11 \\
\hline 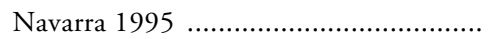 & $-3,45$ & 5,29 & $-8,74$ \\
\hline 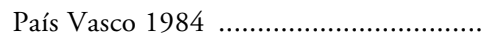 & 3,91 & $-3,78$ & 7,69 \\
\hline 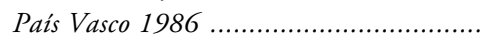 & $-18,3$ & $-6,01$ & $-12,28$ \\
\hline 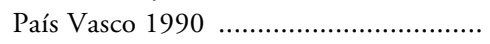 & 2,68 & $-5,15$ & 7,83 \\
\hline 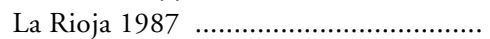 & $-7,22$ & $-9,86$ & 2,64 \\
\hline La Rioja 1995 & $-8,39$ & $-3,32$ & $-5,06$ \\
\hline Comunidad Valenciana 1987 ................. & $-10,09$ & $-5,80$ & $-4,28$ \\
\hline Comunidad Valenciana 1991 ..................... & 1,53 & $-4,46$ & 5,99 \\
\hline Comunidad Valenciana 1995 ................. & $-9,02$ & $-5,24$ & $-3,77$ \\
\hline
\end{tabular}


Sin embargo, esta interpretación es muy sensible a la consideración o no de los outliers a los que ya hemos hecho referencia. Toda vez que son excluidos, los parámetros estimados y la bondad del ajuste convergen considerablemente: ahora el $\mathrm{R}^{2}$ para las Comunidades con competencias amplias [columna (5)] es de 0,46 y para las del artículo 143 de 0,51; mientras que antes de la exclusión de los outliers eran de 0,28 y 0,52, respectivamente. En definitiva, no podríamos rechazar estadísticamente un comportamiento similar en ambos grupos.

Las cosas se nos aparecen distintas al comparar submuestras temporales. En los años ochenta, las variables económico-presupuestarias no habrían sido relevantes, incorporemos [columna (8)] o no [columna (9)] las observaciones anómalas. Por lo tanto, el notable coeficiente de determinación obtenido - 0,60 y 0,66 , respectivamente - vendría explicado por la fuerte dependencia de los resultados autonómicos respecto a los estatales.

En los años noventa, la importancia de los factores internos habría aumentado sensiblemente, tanto cuando se incluyen los outliers [columna (10)] como cuando no [columna (11)]. Este hecho tiene mucho que ver, sin duda, con la progresiva institucionalización de las Comunidades Autónomas (Mota, 1998).

\section{CONCLUSIONES}

La trayectoria de los apoyos electorales de los partidos o coaliciones de gobierno autonómicos en el período 1980-1997 aparece débilmente ligada a la suerte de la gestión gubernamental, cifrada en la evolución de las variables económico-presupuestarias. Sólo los niveles de la inversión pública y, sobre todo, los de paro resultan significativos, aunque de un modo más modesto que el destacado en la literatura anglosajona.

A nuestro juicio, la minusvaloración de las variables económico-presupuestarias autonómicas encontraría su explicación en la propia debilidad de los requisitos políticos que posibilitan la interacción entre economía y política en los sistemas de partidos de las Comunidades Autónomas. Pero también en las particularidades de la descentralización del Estado español: limitada corresponsabilidad y autonomía fiscal, así como protagonismo secundario de numerosas haciendas autonómicas en comparación a las decisiones públicas tomadas en el ámbito estatal y local, hasta hace bien poco.

En este sentido, la evolución paralela de los apoyos electorales autonómicos y estatales refleja perfectamente la dependencia entre ambos ámbitos institucionales: la trayectoria de los partidos o coaliciones de gobierno autonómicos en las elecciones generales es la variable más relevante en nuestro análisis. La lógica político-electoral de las elecciones autonómicas resulta así deudora de lo que acontece en el nivel central, lo que no hace sino subrayar su "rango secundario». En todo caso, la progresiva institucionalización de las Comunida- 
des Autónomas ha llevado a que la coyuntura específica de cada una de ellas haya adquirido mayor relevancia en el pasado reciente: la incidencia de las variables económico-presupuestarias en las arenas autonómicas es más importante en la década de los noventa que en la de los ochenta, al contrario de lo que ocurre con la variable que recoge la dinámica político-electoral estatal.

En cualquier caso, todo esto no implica la inexistencia de escenarios diferenciados o pautas de comportamiento propias derivadas de los contextos autonómicos. La moderada bondad del ajuste econométrico lograda por el modelo habla bien a las claras de la importancia de los elementos específicamente políticos de cada Comunidad Autónoma, que no por ello llevan a comportamientos de voto dual en todos los casos (Pallarés, 1995).

De este modo, además de avanzar en el estudio de las identidades duales en algunas Comunidades Autónomas, el análisis comparado del comportamiento electoral autonómico debe comprender variables específicamente políticas, entre las que el liderazgo se perfila como una de las más importantes. No obstante, la indisponibilidad de datos sobre este particular para la serie completa de elecciones autonómicas celebradas desde 1980 dificulta sobremanera tal aproximación ${ }^{13}$.

En definitiva, el estudio de la evolución de los apoyos electorales de los gobiernos autonómicos pone de manifiesto que las variables políticas tanto estatales como autonómicas cuentan, y mucho, en las diferentes arenas de competición partidista en España: en el pasado, las motivaciones políticas han sido más relevantes que las pautas de racionalidad económica para el comportamiento electoral autonómico.

\section{REFERENCIAS BIBLIOGRÁFICAS}

Alcántara, M., y Martínez, A. (1998) (eds.): Las elecciones autonómicas en España, 19801997, Madrid, Centro de Investigaciones Sociológicas.

Alesina, A. (1988): «Macroeconomics and parties», en S. Fisher (ed.), Macroeconomics Annual 1988, Cambridge, MA, MIT Press.

Alesina, A.; Londregan, J., y Rosenthal, H. (1993): «A model of the Political Economy of the United States", American Political Science Review, 87, pp. 12-33.

Alesina, A., y Rosenthal, H. (1995): Partisan Politics, Divided Governments and the Economy, Cambridge, Cambridge University Press.

Bellucci, M. (1984): «The Effect of Agregate Economics Conditions on the Political Preferences of the Italian Electorate, 1953-1979", European Journal of Political Research, 12, pp. 387-401.

BlaOs, A., y Nadeu, R. (1992): «The Electoral Budget Cycle», Public Choice, 74, pp. 389-403.

BoIX, C. (1996): Partidos politicos, crecimiento e igualdad. Estrategias económicas conservadoras y socialdemócratas en la economía mundial, Madrid, Alianza Universidad.

${ }_{13}$ En el que hasta el momento es el estudio más dilatado en el tiempo sobre la cultura política y la opinión pública en las Comunidades Autónomas (Mota, 1998), sólo se recoge información sobre el liderazgo a partir de 1986, y no para todos los casos. 
ÇARCOĞLU, A. (1995): «Elections manifestos and policy-oriented voting. A pooled cross-national analysis», European Journal of Political Research, 27, pp. 293-317.

Díaz de SARRALDe, S. (1999): «La modelización de los ciclos político-económicos», comunicación presentada al VI Encuentro de Economía Pública, Oviedo, febrero.

ERIKSON, R. (1989): «Economic Conditions and the Presidencial Vote», American Political Science Review, 83, pp. 67-79.

Fuentes, E., y AlCAide, J. (1995): «Los males de la inflación y el déficit público de la economía española», Cuadernos de Información Económica, 100, pp. 3-20.

Greene, W. H. (1997): Econometric analysis, 3. ${ }^{\mathrm{a}}$ ed., Nueva Jersey, Prentice Hall.

Hibbs, D. A. (1992): «Partisan theory after fifteen years», European Journal of Political Economy, 8, pp. 361-373.

LAGO, S. (1997): "¿Podemos falar de ciclo orzamentario electoral no caso dos municipios galegos?", Revista Galega de Economía, 6, pp. 259-270.

LEWIS-BECK, M., y BELlUCCI, P. (1982), «Economic Influences on Legislative Elections in Multiparty Systems: France and Italy», Political Behavior, 4, pp. 93-107.

LlerA, F. J. (1998): «Los rendimientos de los sistemas electorales de las Comunidades Autónomas: el predominio del bipartidismo imperfecto», REIS, 82, pp. 127-157.

Maravall, J. M., y Fraile, M. (1998): The politics of unemployment: the Spanish experience in comparative perspective, Madrid, Centro de Estudios Avanzados en Ciencias Sociales.

Monroe, K. R. (1979): «Economic Analysis of Electoral Behavior: A Critical Review», Political Behavior, 1, pp. 137-173.

Montero, J. R., y PALlarés, F. (1992): Los estudios electorales en España: un balance bibliográfico (1977-1992), Barcelona, Institut de Ciències Politiques i Socials, Working Paper.

Mota, F. (1998): Cultura política y opinión pública en las Comunidades Autónomas: un examen del sistema politico autonómico en España 1984-1986, Barcelona, Institut de Ciències Politiques i Socials, Working Paper.

Paldam, M. (1981): "A Preliminary Survey of the Theries and Findings on Vote and Popularity Functions", European Journal of Political Research, 9, pp. 181-199.

PAllarés, F. (1995): “Las elecciones autonómicas en España: 1980-1992», en P. del Castillo (ed.), Comportamiento politico y electoral, Madrid, Centro de Investigaciones Sociológicas, pp. 151-220

Pallarés, F.; Montero, J. R., y Llera, F. J. (1997): «Non State-Wide Parties in Spain: An Attitudinal Study of Nationalism and Regionalism», Publius; The Journal of Federalism, 27, pp. 135-169.

Powell, G. A., y Whitten, G. D. (1993): «A cross-national analysis of the economic voting: Taking account of the political context», American Journal of Political Science, 97, pp. 391414 .

RAYMOND, J. L. (1994): "Condicionantes externos de la evolución de la economía española», Documentos de Trabajo, núm. 104, Fundación FIES.

SAnjiaO, F. (1991): «Una aproximación al estudio de los ciclos político económicos», en P. Puy (coord.), Estudios en homenaje al profesor Carlos Otero Díaz, Santiago de Compostela, Universidad de Santiago de Compostela, pp. 651-673.

SuÁrez Pandiello, J. (1996), "Las Comunidades Autónomas y su endeudamiento: un test sobre sus causas», en E. Moldes y P. Puy (eds.), La financiación de las Comunidades Autónomas, Madrid, Minerva Ediciones, pp. 185-210.

WERT, I. (1998): «Elecciones autonómicas en España, 1980-1996: una visión de conjunto», en M. Alcántara y A. Martínez (eds.), Las elecciones autonómicas en España, 1980-1997, Madrid, Centro de Investigaciones Sociológicas, pp. 503-525.

Whitten, G. D., y Palmer, H. D. (1999): «Cross-National Analyses of Economic Voting», Electoral Studies, 18, pp. 49-67. 


\section{ABSTRACT}

This article aims to study the validity of incumbency-oriented hypothesis in the Spanish autonomous regions. The results of our analysis suggest that the political factors are more relevant than the evolution of economic and budget variables in explaining and predicting electoral support given to the parties in government. 


\section{TEXTO CLÁSICO}

